\title{
V. Rückkehr nach Europa
}

Trotz Schauffs Skepsis, daß auf zurückkehrende Emigranten sogleich „staatspolitische Aufgaben" warteten ${ }^{1}$, verstärkte sich mit diesen Aktivitäten der innere Drang einer Rückkehr nach Europa, um dort, vor Ort, helfend in das Geschehen eingreifen zu können, aber auch, um politisch wieder präsent zu sein oder doch die Möglichkeit politischer Betätigung selbst auszuloten. Stark war auch das Bedürfnis, nach alten politischen Freunden zu forschen und festzustellen, ob sie den Terror überlebt hatten. Dazu kam die Überzeugung, daß trotz allen Elends und der Zerstörungen unter den Trümmern noch viele Werte weiter vorhanden seien, die die Eltern Schauff und ihre Kinder in der Neuen Welt hatten entbehren müssen². Indes war die Rückkehr nicht ohne Hindernisse.

Innerhalb Brasiliens durfte Schauff reisen - wenn auch nur mit Sondergenehmigungen. Als er sich 1948 anschickte, das erste Mal außer Landes zu gehen, hatte allerdings - in Brasilien wie im übrigen Lateinamerika - der amerikanische Botschafter das letzte Wort, wenn es um die Rückwanderung von politischen Emigranten nach Deutschland ging. Wie in zahlreichen anderen Fällen waren auch im Falle Schauff amerikanische Dienststellen für anonyme Einflüsterungen und Denunziationen empfänglich, deren Urheber konservative Emigranten einer genuinen Komplizenschaft mit den Nationalsozialisten beschuldigten. Als Schauff sich um ein Reisevisum beim US-Konsulat in São Paulo bemühte, wurde er vom Vizekonsul mit einem "file" konfrontiert, aus dem hervorging, daß er bis 1940 Mitglied des Reichstags und somit ein „Nazi“ gewesen sei. Diese Affäre, die von April bis Oktober 1948 andauerte und Schauff zunächst die Bewilligung eines amerikanischen Visums kostete, konnte schließlich bereinigt werden ${ }^{3}$. Schauff erhielt durch die brasilianischen Behörden einen sogenannten Fremdenpaß; seine definitive Einbürgerung sollte erst 1950 erfolgen ${ }^{4}$. Noch im gleichen Jahr 1948 flog er von Rio de Janeiro nach Rom.

Dort traf Schauff zuerst auf Ludwig Kaas - jenen ehemaligen Reichstagskollegen, der der Familie bereits in den römischen Exiljahren zur Seite gestanden hatte. Kaas, Vertrauter des Kardinalstaatssekretärs und späteren Papstes Pius XII., war seit 1936 verantwortlicher ökonomischer Leiter der Basilika Sankt Peter und in dieser Funktion auch mit archäologischen Forschungen unter der Peterskirche

1 Schreiben Johannes Schauff an den ebenfalls emigrierten Gründer der "Volkssozialistischen Bewegung“, Hans Jaeger (BHB I), 21. August 1946 (IfZ, NL Schauff, Bd. 34).

2 So Karin Schauff in: Um der Freiheit willen, S. 30.

3 Korrespondenz mit US-Botschaft, 21. April-27. Oktober 1948; Protokoll eines Gesprächs Johannes Schauff mit dem US-Konsul John B. Ocheltree (IfZ, NL Schauff, Bd. 10).

4 IfZ, NL Schauff, Bd. 27. Innerhalb Brasiliens war bis dahin nach Schauffs Aussage ein Ersatzdokument der Companhia de Terras Norte do Paraná bei Behörden und Regierungsstellen von außerordentlicher Effizienz (die Kopie eines solchen Dokuments in IfZ, NL Schauff, Bd. 27, datiert vom 10. April 1943). 
befaßt ${ }^{5}$. Während der Herrschaft des Faschismus in Italien und vor allem unter der deutschen Besetzung Roms hatte sich Kaas zunehmend unsicher und bedroht gefühlt ${ }^{6}$ und war unterdessen „ein geprüfter und leidgereifter Mann"geworden ${ }^{7}$.

Die durch den Krieg ausgelöste Flüchtlingswelle bereitete Kaas ebenso große Sorgen wie Johannes Schauff. Bereits Ende 1946 hatte eine Delegation des Vatikans Schauff auf seiner brasilianischen Fazenda besucht, um Immigrationsmöglichkeiten auszuloten ${ }^{8}$. Während Schauffs Aufenthalt in Rom konnte er mit Kaas in dieser Frage auch bei Pius XII. vorsprechen, der Schauff bereits bei dieser ersten Begegnung darum bat, sich für die Bewältigung des Flüchtlingsproblems zur Verfügung zu stellen. Dies kam Schauffs Intentionen durchaus entgegen ${ }^{9}$. Erste organisatorische Überlegungen wurden zusammen mit Vertretern der amerikanischen und internationalen Caritas angestellt, die sich zu dieser Zeit ebenfalls in Rom befanden: James J. Norris, seit 1947 Direktor der europäischen Sektion der Catholic Relief Services, und Monsignore Luigi Ligutti, Exekutivdirektor der National Catholic Rural Life Conference.

Bemühungen, von Italien aus auch Deutschland zu besuchen, scheiterten an den Besatzungsverwaltungen, die Schauff keine Einreisepapiere auszustellen bereit waren. Erster Begegnungsort mit politischen Freunden aus Deutschland wurde daher die Schweiz, in die einzureisen Schauff keine Schwierigkeiten hatte.

Die Schweiz war - neben den USA und Lateinamerika sowie England und der Türkei - ein Zentrum der bürgerlichen und konservativen Emigration. Hier lebte der ehemalige Reichskanzler Joseph Wirth, der - zusammen mit seinem Paladin, dem Schriftsteller Jakob Kindt-Kiefer ${ }^{10}$ - in der Gruppe "Demokratisches Deutschland“ aktiv war. Zur Exilbewegung „Freies Deutschland"11 gehörten der katholische Publizist Franz Albert Kramer und der protestantische Theologe Siegmund-Schultze ${ }^{12}$, die die Organisation nach deren Majorisierung durch die Kommunisten allerdings wieder verließen. Auch Wilhelm Röpke, der 1933 zu-

${ }^{5}$ Die Ausgrabungen unter der Basilika führten 1950 zur Auffindung des Grabes des Apostels Petrus.

6 Vgl. Karin Schauff, Ludwig Kaas, S. 15 f.

7 May, Ludwig Kaas, Bd. III, S. 451.

${ }^{8}$ Brief Johannes Schauff an Hermann Dietrich (ehemals Reichsminister in den Kabinetten Müller und Brüning, nach 1945 Vorsitzender des Ernährungs- und Landwirtschaftsrats der US- und der britischen Besatzungszone) vom 15. Februar 1947: Das Problem werde von Tag zu Tag dringlicher, „aber leider fehlt sowohl den Auswanderungs- wie den Einwanderungsländern jede Großzügigkeit in der Behandlung dieser Frage“ (IfZ, NL Schauff, Bd. 32).

9 Er wolle nur nach Europa zurückkehren, schrieb er am 18. Juli 1946 an Karl Thieme, wenn er auf seinem „Fachgebiet der Agrarreform, Kolonisation und Auswanderung Positives leisten" könne. "Leider geschieht auf diesem Gebiet meines Erachtens östlich der Elbe zuviel, westlich zu wenig. Zwar hoffe ich, daß die Welt bald einsehen wird, daß Zentraleuropa Menschen an die übrigen Kontinente abgeben muß. Dann wäre mein Platz in der internationalen Wanderungsbewegung, in der ich aber nicht als Lakai zu arbeiten gedenke." (IfZ, NL Schauff, Bd. 8).

10 Biographie Jakob Kindt-Kiefer in BHB I; siehe auch S. 132, Anm. 3.

11 Vgl. Bergmann, Freies Deutschland.

12 Friedrich Siegmund-Schultze (1885-1969), Hochschullehrer und Sozialpädagoge, kehrte 1946 nach Deutschland zurück (BHB I). 
nächst in die Türkei emigriert war, befand sich ab 1937 in der Schweiz und lehrte am Institut des Hautes Etudes in Genf ${ }^{13}$. Die Schweiz bildete darüber hinaus nach Kriegsende eine wichtige Zwischenstation für eine Rückkehr nach Deutschland und war der Ort, an dem erste Begegnungen von Emigration und innerem Widerstand stattfanden.

In einem Hotel in Luzern traf Schauff nach langen Jahren auch Joseph Wirth wieder, seinen früheren politischen Mentor. Zu einem ersten Gedankenaustausch kam er auch mit seinem alten, ebenfalls rheinisch-katholisch geprägten politischen Weggefährten Franz Albert Kramer zusammen, der als Lizenträger des Rheinischen Merkur in Westdeutschland politisch einflußreich geworden war ${ }^{14}$. Er traf auf Karl Thieme und Karl Fütterer ${ }^{15}$ sowie auf weitere Männer des inneren Widerstands wie den ehemaligen Präses der katholischen Arbeitervereine, Hermann Joseph Schmitt, der ihm aus seiner Haft im Konzentrationslager berichtete ${ }^{16}$.

Als Schauff drei Monate später von dieser ersten Informationsreise in Europa nach Brasilien zurückkehrte, stand für ihn angesichts der politischen Schwierigkeiten und der materiellen Not heimatlos gewordener Bevölkerungsteile in Europa die Entscheidung zur Rückkehr fest. Bereitschaft und Drang, vor Ort tätig zu werden, hatten Schauff von jeher ausgezeichnet; seine eigenen Erfahrungen von Widerstand, Verfolgung und Emigration trachtete er in einen Versuch der Hilfe zur Selbsthilfe einzubringen.

Der wirtschaftliche Erfolg der Fazenda in Brasilien, dessen Grundlage die weltweit günstige Kaffeekonjunktur war, erleicherte die Entscheidung zur Rückkehr.

13 Wilhelm Röpke (1899-1966), 1928 Professor für politische Ökonomie in Graz, 1929 in Marburg; offener NS-Gegner, als Hochschullehrer entlassen; 1933 Emigration in die Türkei, Professor an der Universität Istanbul, dort mit Alexander Rüstow und Fritz Neumark Aufbau der wirtschaftswissenschaftlichen Fakultät. 1937 Berufung nach Genf. Nach 1945 einer der Theoretiker der „sozialen Marktwirtschaft“. Röpke war Vertreter eines wirtschaftlichen Liberalismus von weltanschaulich konservativer Prägung und Föderalist. Er hatte bereits von 1945 bis 1947 mit Johannes Schauff korrespondiert und die gesellschaftliche und politische Entwicklung in Deutschland erörtert. In diesem Zusammenhang findet sich Schauffs Vorschlag einer Neuauflage der "Notgemeinschaft deutscher Wissenschaftler im Ausland“, IfZ, NL Schauff, Bd. 6. (Die Biographien von Röpke, Rüstow und Neumark in BHB II).

14 Franz Albert Kramer (gest. 1950), Mitarbeiter und Korrespondent der Kölnischen Volkszeitung und des Zentrums-Blattes Germania, erhielt nach 1933 Berufsverbot. Nach seiner Emigration war er journalistisch in Paris, London und Rom tätig, wurde auf deutsche Intervention aus Italien ausgewiesen und emigrierte in die Schweiz, in contumaciam zum Tode verurteilt. Kramer erhielt sehr früh die Lizenz für eine Zeitungsgründung, so daß am 15. März 1946 die erste Ausgabe des Rheinischen Merkur erscheinen konnte. Vgl. Boll/ Schulze/Süssmuth, Zeitungsland Nordrhein-Westfalen, S. $443 \mathrm{ff}$.; Schneider, Christliche und konservative Remigranten, S. $167 \mathrm{ff}$. Zum politischen Einfluß des Rheinischen Merkur vgl. Schwarz, Vom Reich zur Bundesrepublik, S. 394 ff., bes. S. 421 f.; siehe auch Kap. XIII, S. $211 \mathrm{f}$.

15 Karl Fütterer, ein Bekannter Schauffs aus der Siedlungsbewegung, hatte zu christlichen Widerstandskreisen im Rheinland gehört. Siehe oben, S. 69.

16 Schmitt stand in Verbindung zu Kreisen des 20. Juli 1944 (Alfred Delp, Nikolaus Groß und Bernhard Letterhaus - vgl. oben, S. 68, Anm. 25), war ab Dezember 1944 im KL Dachau inhaftiert, dort vorübergehend in Bunkerhaft; Flucht während des Evakuierungsmarsches (Ende April 1945). 
Die Ländereien in Schauffs Besitz hatten im Laufe der Jahre immens an Wert gewonnen, wobei das während des Krieges durch die brasilianischen Behörden ausgesprochene Verkaufsverbot für Grundbesitz von deutschen Siedlern sich nach 1945 finanziell schließlich ausgesprochen positiv auswirkte. Die Farm machte Schauff auch bei einer Rückkehr nach Europa materiell unabhängig.

An dieser Stelle sei noch einmal auf Schauffs organisatorische Begabung und seine profunde Kenntnis des bäuerlichen Wirtschaftens und Siedelns hingewiesen, die solche wirtschaftliche Erfolge erst ermöglichten. In Rolândia waren zwar auch andere Siedler erfolgreich, deren bürgerliche Berufe sie zu solchen Unternehmungen an sich nicht prädestiniert hatten ${ }^{17}$. Doch scheint Schauff mit seinen spezifischen Talenten in dieser Gruppe ein Primus inter pares gewesen zu sein ${ }^{18}$.

Bevor die Familie 1951 definitiv nach Europa zurückkehrte, brach Johannes Schauff im Frühjahr 1949 zu einer weiteren Tour d'horizon auf den alten Kontinent auf, um Klarheit über zwei mögliche Optionen seines Engagements zu gewinnen: Dies waren die deutsche Nachkriegspolitik und die internationale Flüchtlingsarbeit. Bei dieser zweiten Europareise konnte Schauff Deutschland besuchen: Seine Einreise in die französische Besatzungszone wurde durch eine Einladung des Freiburger Universitätsprofessors Constantin von Dietze ermöglicht, der in der Weimarer Republik zu den engagierten Verteidigern der inneren Kolonisation gehört hatte ${ }^{19}$. Zum Zeitpunkt dieser Reise stand die Gründung der Bundesrepublik unmittelbar bevor. Schauff konnte mit alten politischen Freunden und Weggefährten aus der Zeit der Windthorstbewegung wie Heinrich Krone und Hermann Kopf zusammentreffen.

Krone hatte zum katholischen Widerstand gegen die nationalsozialistische Diktatur gehört. Eines der inhaltlichen Ziele dieser Widerstandskreise war eine große, konservative christliche Volkspartei, nachdem das Zentrum politisch gescheitert war ${ }^{20}$. Die Politiker, die Schauff in diesem Umfeld antraf, gehörten zu den Weg-

17 So etwa der Frankfurter Rechtsanwalt Max Hermann Maier (vgl. oben, S. 82, Anm. 46).

18 Dies läßt sich etwa aus dem Beitrag von Wolfgang Hoffmann-Harnisch (Regisseur und Autor, ab 1938 im brasilianischen Exil, 1951 Rückkehr nach Deutschland) zu dem von Schauff hrsg. Band: Landerschließung, S. 6, ableiten.

19 Constantin von Dietze (1891-1973), ab 1937 o. Prof. an der Universität Freiburg, Agrarwissenschaftler; ab 1951 Herausgeber der Zeitschrift Innere Kolonisation (vgl. Boyens, Siedlung, Bd. II, S. 29 und 209ff.). Dietze gehörte dem am 11. September 1950 unter dem Vorsitz von Bundespräsident Theodor Heuss konstituierten ersten Wissenschaftlichen Beirat des „Deutschen Instituts für Geschichte der nationalsozialistischen Zeit" an (ab 1952 Institut für Zeitgeschichte).

20 Siehe Kap. II/2, S. 57 f.; Winfried Becker, Politische Neuordnung aus der Erfahrung des Widerstands: Katholizismus und Union, in: Steinbach (Hrsg.), Widerstand., S. 261-292. Walther Hensel, einer der Exponenten des rheinischen katholischen Widerstands (siehe oben, S. 67), berichtet, daß die „Idee einer die positiven katholischen und evangelischen Christen umfassenden Partei“ in dem Widerstandskreis, dem er angehörte, "wohl erstmals um die Mitte des Jahres 1934 erörtert worden“ sei. Der Wille zur Gründung einer überkonfessionellen Partei sei nicht nur aus dem „Gedanken einer Vereinigung der Kräfte zum Zwecke der Abwehr" entstanden, sondern habe vielmehr in der Überzeugung gewurzelt, „daß die Entchristlichung des Lebens in Deutschland ursächlich gewesen“ sei „für die Entartung unseres politischen Lebens ... und daß nur eine Partei, die von den Grundsätzen des Christentums getragen" sei, auch "die notwendige Erneuerung und Gesundung“ 
bereitern der Christlich-Demokratischen Union. Deren herausragende Gestalt und erster Kanzler der jungen Bundesrepublik, Konrad Adenauer, war schließlich in Bonn ein wichtiger Gesprächspartner in Hinblick auf Schauffs Rückkehr in die deutsche Politik.

Mit Konrad Adenauer verband Schauff die rheinisch-katholische Prägung und es bestanden durchaus persönliche und freundliche Beziehungen. Dies illustriert eine Begegnung in den sechziger Jahren, an der auch Heinrich von Brentano teilnahm und bei der u.a. die Frage debattiert wurde, wer von beiden - Adenauer oder Schauff - die meisten Kinder und Enkelkinder habe. Dabei wurde offenbar, daß der um 27 Jahre jüngere Schauff deutlich mehr Enkelkinder vorweisen konnte als Adenauer (die Familie Schauff hatte zu dieser Zeit bereits 40 Enkel, Adenauer zu Lebzeiten deren 24). Schauff führte dieses ungleiche Zahlenverhältnis darauf zurück, daß sich unter Adenauers Kindern ein Geistlicher befinde, worauf Adenauer bestätigte: „Dat stimmb, dä deeth nit mit.“ Adenauer führte seinerseits an, daß Schauff ja Z willingssöhne habe, worauf dieser auf den aus seiner Sicht „unlauteren Wettbewerb" hinwies, weil Adenauer ja zweimal verheiratet gewesen sei. Der edle Wettstreit sei mit einer guten Flasche Wein beschlossen worden ${ }^{21}$.

$\mathrm{Daß}$ aus einem Amt in der neuen Regierung dann doch nichts wurde - Schauff hatte zuletzt auch die Offerte abgelehnt, als Botschafter nach Brasilien zu gehen -, lag wohl vor allem an Unterschieden in politischer Haltung und Entwicklung von Adenauer und Schauff. Daß in der CDU Remigranten so gut wie keine Rolle spielten, hatte sehr viel mit Vorurteilen auf beiden Seiten zu tun - das Exil war ein schwer überbrückbarer Graben. Dies gilt für Heinrich Brüning, aber auch für den politisch so unglücklich agierenden Joseph Wirth'22, den Schauff zuvor wieder in Freiburg getroffen hatte und dem er sich solidarisch verbunden fühlte, ebenso für Andreas Hermes ${ }^{23}$, Carl Spiecker ${ }^{24}$ und Reinhold Treviranus ${ }^{25}$, der im Exil zum

der Gesellschaft und der Politik bewirken könne (Walther Hensel in einem Brief vom 5. Mai 1952 an den damaligen Ministerpräsidenten von Nordrhein-Westfalen, Karl Arnold, der ebenfalls zum rheinischen Widerstand gehört hatte; vgl. auch Zeugenschrifttum Walther Hensel, IfZ, ZS 534).

21 Diese Adenauer-Anekdote, die der Kölner Zeitungsverleger Heinrich Heinen (Kölner Rundschau, Bonner Rundschau) bei einem Gespräch mit Schauff am 10. September 1975 aufzeichnete, erschien in der Silvesternummer der Bonner Rundschau, 1. Januar 1976; die diesbezügliche Korrespondenz Schauff-Heinen in IfZ, NL Schauff, Bd. 3.

22 Vgl. Morsey, Leben und Überleben im Exil, S. 88ff.; Schwarz, Adenauer. Der Aufstieg, S. 642 ff.; Küppers, Wirth, S. 301-328; Hörster-Philipps, Wirth, S. 714 ff.

23 Andreas Hermes (1879-1964), Zentrumspolitiker, 1920-22 Reichsminister für Ernährung, 1922-23 Reichsfinanzminister im Kabinett Cuno, 1924-29 Mitglied des Preußischen Landtags, 1928-33 MdR., Agrarpolitiker, Präsident der Deutschen Bauernvereine und des Raiffeisenverbandes. Nach 1933 politisch verfolgt und inhaftiert. 1936 Emigration nach Kolumbien, Aufbau eines ländlichen Genossenschaftswesens. März 1939 Deutschlandbesuch, vom Kriegsausbruch überrascht, Anschluß an Widerstandskreis um Carl Goerdeler, 20. Juli 1944 verhaftet und am 11. Januar 1945 zum Tode verurteilt. Nach der Befreiung aus der Vollstreckungshaft Mitbegründer und Vorsitzender der CDU in der SBZ, von der sowjetischen Besatzungsmacht zum Rücktritt gezwungen, Übersiedlung nach Bad Godesberg. 1946-54 Vorsitzender des wiedergegründeten Bauernverbandes und 1947-61 Vorsitzender des Deutschen Raiffeisenverbands; Mitglied des Frankfurter Wirtschaftsrates (BHB I).

24 Carl Spiecker (1888-1953), Journalist, Redakteur der Zentrums-Presse, 1922-23 Verlags- 
Kreis um Heinrich Brüning gehört hatte. Ohne politische Fortüne blieb schließlich auch Schauffs Weggefährte aus der Weimarer Siedlungsbewegung, Hans Schlange-Schöningen, der zu den Wegbereitern der Siedlung auch in Brasilien gehört hatte und dennoch nicht emigriert war, sondern sich statt dessen dem Widerstand angeschlossen hatte ${ }^{26}$.

Schauff gewann damals den Eindruck, daß Adenauer beim staatlichen Neuaufbau allzu sehr auf Vertreter der alten bürgerlichen Eliten setzte, die in seinen Augen durch den Nationalsozialismus diskreditiert seien: „Im neuen Auswärtigen Amt" - so schrieb er 1950 enttäuscht an den Jesuitenpater Robert Leiber „,regieren' die Korpsstudenten schon wieder." ${ }^{27}$ Schauff, der auf seiner zweiten Europareise auch mit Kurt Schumacher zusammentraf, war dagegen überzeugt, daß nach dem vorangegangenen politischen Desaster und den Gründen, die dazu geführt hatten, die Sozialdemokraten bei der Neubildung des Staates nicht ausgeschlossen werden dürften.

Schauffs Vorbehalte gegen die Übernahme eines Regierungsamtes in Deutschland hatten darüber hinaus ihren Grund aber auch in der stark eingeschränkten Souveränität und Bewegungsfreiheit der neuen deutschen Regierung. Allzu drükkend und ohne absehbares Ende erschien ihm bei diesen ersten Besuchen das Gewicht der alliierten Militärregierungen. $\mathrm{Zu}$ fragen ist, ob hinter dieser negativen Bestandsaufnahme nicht auch eine im Exil vollzogene Abkehr von nationalstaatlichem Denken stand, wie sie auch Koch-Weser in Rolândia geistig vollzogen hatte. So mochte für den inzwischen zum Weltbürger gewordenen Johannes Schauff die definitive Option für die in Rom offerierte internationale Tätigkeit näherliegend gewesen sein, in deren Rahmen zudem die Möglichkeit gegeben schien, der jungen Republik von außen mehr nützen zu können.

direktor der Germania; Tätigkeit im Auswärtigen Amt und als Leiter der Presseabteilung der Reichsregierung, 1930-31 unter Innenminister Joseph Wirth Sonderbeauftragter zur Bekämpfung der NS-Bewegung. 1933 Emigration nach Frankreich, aktiv in der Volksfrontbewegung. 1937/38 Initiator der "Deutschen Freiheitspartei“ (Exilorganisation liberal-konservativen Zuschnitts, die sich als Auslandsvertretung des innerdeutschen Widerstands verstand und Rückhalt vor allem in Kreisen der Wehrmacht, Kirchen und Wirtschaft hatte; die ab 1937 ins Reich geschleusten „Deutschen Freiheitsbriefe“ betonten die Rolle der Wehrmacht als einzigem Machtfaktor zum Sturze des Regimes, ihr Vertriebsnetz wurde aber 1938 aufgedeckt, es kam zu Hochverratsverfahren gegen Sympathisanten). 1940 nach der Besetzung Frankreichs Flucht nach Großbritannien, Aufbau und Leitung eines „Freiheitssenders der Deutschen Freiheitspartei“; 1941 Emigration nach Kanada und 1945 Rückkehr nach Deutschland. Lizenzträger der Rbein-Rubr-Zeitung in Essen, 1946 beteiligt an Neugründung des Zentrums, 1947 MdL Nordrhein-Westfalen und Mitglied des Wirtschaftsrats in Frankfurt, 1949 CDU, Minister ohne Geschäftsbereich (BHB I).

25 BHB I; Möller, Treviranus, S. $134 \mathrm{ff}$.

26 Hans Schlange-Schöningen (1886-1960) gehörte in der Weimarer Republik zur DNVP, war Mitbegründer der „Volkskonservativen Vereinigung" und hatte später Verbindungen zum Kreisauer Kreis. Nach 1945 gehörte er zu den Mitbegründern der CDU, 1949 MdB, 1950 Generalkonsul und ab 1953 Botschafter in London, 1955 im Ruhestand. Unorthodoxer und aktiver Ostpolitiker. Zum problematischen Verhältnis Adenauer-SchlangeSchöningen vgl. die Korrespondenz Johannes Schauff mit dem Sohn Hans-Joachim Schlange-Schöningen (IfZ, NL Schauff, Bd. 7).

27 Brief Johannes Schauff an Robert Leiber SJ, 6. Februar 1950 (IfZ, NL Schauff, Bd. 14). 